\title{
Study on Synthetic Evaluation of Human Performance in Manually Controlled Spacecraft Rendezvous and Docking Tasks
}

\author{
Ting Jiang, Chunhui Wang*, Zhiqiang Tian, Yongzhong Xu, and Zheng Wang \\ National Laboratory of Human Factors Engineering, \\ Astronaut Research and Training Center of China, Beijing 100094, China \\ chunhui_89@yahoo.com.cn
}

\begin{abstract}
Manual-Control Rendezvous and Docking (MCRVD) is a complex and demanding task which requires astronauts to control the direction of six-freedom of the spacecraft accurately by hand. Its performance has a close relationship among the design of the spacecraft HCI, the control ability of the astronaut, and the matching effect of the two factors above. In this paper, human performance for these tasks was measured and a mathematical model to evaluate MCRVD performance quantitatively was proposed. First, 3500 experiments were designed on a ground simulated RVD system to examine characteristics and regulations of MCRVD performance indexes, such as control deviation, fuel consumption etc. Twenty-five male volunteers aged 25-35 participated in the experiment. Analysis predicts that the performance indexes of MCRVD show the characteristics and laws of stages. The process of MCRVD can be divided into three stages: tracking control (about more than 20m distance), accurate control (less than $20 \mathrm{~m}$ distance) and docking stage $(0 \mathrm{~m})$. The performance indexes of tracking and accurate control show the relevance of characteristics, and the precision index of docking reflects the difficulty of control the direction of the spacecraft. For that reason, several statistics techniques, such as the factor analysis method, the entropy analysis method etc, are utilized to analyze the weight coefficient of each performance index. After that we presented a novel multi-hierarchy integrated evaluation method, which includes four hierarchies: the performance of the tracking control, the performance of the accurate control, the precision of docking and the result of docking. Finally we utilized this method to analyze the human performance in MCRVD, which verifies the validity of our method.
\end{abstract}

Keywords: Manual-control Rendezvous and Docking, Human Performance, evaluation method.

\section{Introduction}

The rendezvous and docking (RVD) process consists of orbital maneuvers and controlled trajectories, which successively bring the active vehicle (chaser) into the

\footnotetext{
* Corresponding author.
} 
vicinity of, and eventually into contact with, the passive vehicle (target). RVD technology and techniques are key elements in missions such as assembly in orbit of large units, re-supply of orbital platforms and stations, exchange of crew, and lunar/planetary mission.[1-6]

The Manual control technique is important in spacecraft rendezvous and docking. The human Performance has a close relationship among the design of the spacecraft HCI, the control ability of the astronaut, and the matching effect of the two factors above. This paper aimed at the human performance model to evaluate the degree of matching.

\section{Manual-Control Rendezvous and Docking}

As shown in Fig. 1, Manual-control Rendezvous and Docking (MCRVD) is a manned system consisted of human, display and control. MCRVD is started from the final phases of rendezvous, about 150 meters. In the phases, astronaut estimate the relative distance and attitude between the spacecrafts depend on the video of target in display and parameters data from measure system. At the same time, astronaut also operates six-axis control of spacecraft.

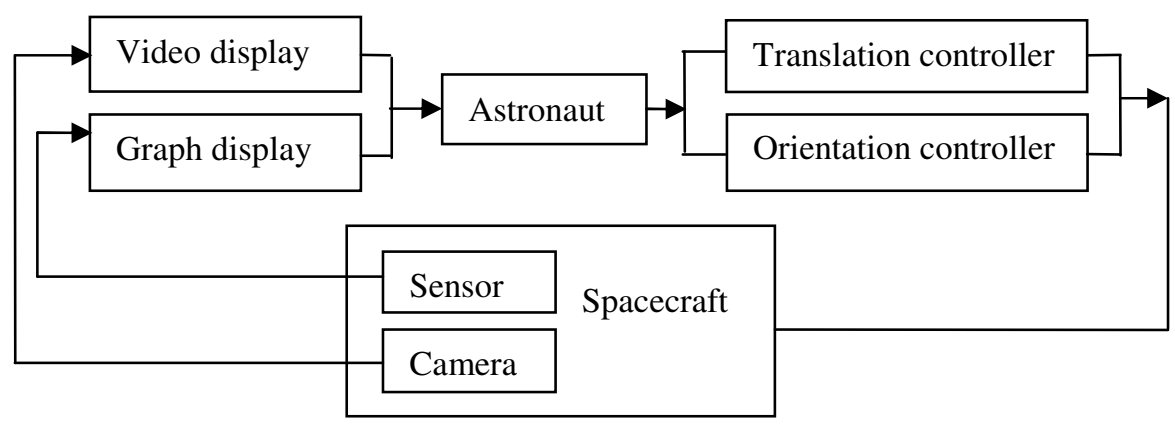

Fig. 1. The human-display-control system in Manual-control Rendezvous and Docking

In Human-display-control system, the performance of task depends on the matching between the parts of system. It is difficult that estimating of the matching of system by a few simple performance indexes. It is important that the system performance model used to evaluate the composite performance by multi-indexes.

\section{Evaluate Index System and Screening Methods}

Performance index system should be constructed to meet the following two principles: On the one hand, it's principle of comprehensiveness, that is, evaluation of the performance index system to reflect all aspects of the object or different properties; On the other hand, it's principle of typicality, that is, a single index must contain the actual physical meaning, to reflect some aspect of evaluation objects or features. For the 
characteristics of MCRVD, this paper through primary and screening to meet the principles of both.

\subsection{Evaluate Index System}

The purpose of primary screen is the selection of indexes as much as possible to describe all aspects of evaluation objects and features, fully meet the principle of comprehensiveness. In this study, Analytic Hierarchy Process (AHP) was used to establish the structure of index system. Through the analysis of indexes properties and physical meaning, the index system was divided into two levels and three categories: Docking results, Docking accuracy and the indexes of Control process, as shown in Table. 1.

Table 1. Evaluate index system

\begin{tabular}{cc}
\hline Level 1 & Level 2 \\
\hline Docking results & Success rate \\
Docking accuracy & Docking deviation \\
& Docking Velocity \\
& Control time \\
Indexes of Control process & Fuel consuming content \\
& Control deviation \\
& Alignment hold rate \\
& Velocity \\
\hline
\end{tabular}

Docking deviation contain that: Translation distance deviation, roll angular deviation, pitch angular deviation and yaw angular deviation. Docking Velocity contain that: X velocity, Translation velocity, roll angular velocity, pitch angular velocity and yaw angular velocity.

Control time is the time from the beginning of manual control to the contact between the two spacecrafts. Fuel consuming content is the sum of fuel in the whole manual control Rendezvous and Docking. The indexes of Control deviation contain the total deviation, average deviation and standard deviation of $\mathrm{Y}$ distance deviation, $\mathrm{Z}$ distance deviation, Translation distance deviation, roll angular deviation, pitch angular deviation and yaw angular deviation. The indexes of Velocity contain the maximum velocity and average velocity of $\mathrm{X}$ velocity, $\mathrm{Y}$ velocity, $\mathrm{Z}$ velocity, roll angular velocity, pitch angular velocity and yaw angular velocity.

\subsection{Screening Method}

Each index of performance indexes system should have a specific physical meaning, can reflect the differences in operating characteristics. In this study, some coefficients of variation were used to measure the degree of difference, e.g. mean and standard deviation. The greater the value of variability, then the higher the discrimination of index, on the contrary, that index is not strong discriminability. This method was used to screen the indexes of docking accuracy. 
In the index system, especially within all levels, indexes have large overlap between the information. So that, it is have a need for indexes screening to keep the representative, as far as possible. Correlation coefficient was used to screen the indexes of rendezvous process.

According to the analysis of data, the Control process was divided into two parts, tracking control stage and accurate control stage. Tracking control stage is about from the start distance to $20 \mathrm{~m}$, Owing to the far distance of target, detailed features can' $\mathrm{t}$ be observed, astronaut mainly track the target by observing the shape of features. Accurate control stage is form $20 \mathrm{~m}$ to $0 \mathrm{~m}$, astronaut mainly precise control the attitude and translation of vehicle to meet the docking access. The screen indexes of both stages are shown in Table.2.

Table 2. The indexes of control process

\begin{tabular}{ccc}
\hline Indexes & $\begin{array}{c}\text { Tracking control } \\
\text { stage }\end{array}$ & $\begin{array}{c}\text { Accurate control } \\
\text { stage }\end{array}$ \\
\hline Control time & $\sqrt{ }$ & $\sqrt{ }$ \\
Fuel consuming content & $\sqrt{ }$ & $\sqrt{ }$ \\
Alignment hold rate & $\sqrt{ }$ & $\sqrt{ }$ \\
Total roll angular deviation & $\sqrt{ }$ & \\
Total translation distance & $\sqrt{ }$ \\
deviation & $\sqrt{ }$ & $\sqrt{ }$ \\
Total gesture angular deviation & $\sqrt{ }$ & $\sqrt{ }$ \\
Gesture alignment hold rate & $\sqrt{ }$ \\
Maximum X velocity & $\sqrt{ }$ & \\
Average X velocity & & \\
Average translation velocity & &
\end{tabular}

\section{Synthetic Evaluate Model}

\subsection{The First Step of Performance Model}

The first step of performance model was :

$$
S=F_{1} *\left(w_{2} * F_{2}+w_{3} * F_{3}\right)
$$

Where $S$ is the value of performance, $F_{1}, F_{2}$ and $F_{3}$ are the values of Docking results, Docking accuracy and the indexes of Control process, $w_{2}$ and $w_{3}$ are the weight coefficient of Docking accuracy and the indexes of Control process.

The weight coefficient of Docking accuracy and the indexes of Control process were analyzed by expert assign method. 


\subsection{The Second Step of Performance Model}

The second step of performance model has two parts: the model for evaluates docking precision and the model for evaluates Rendezvous process.

Docking precision contain offset precision and velocity precision. The model was:

$$
F_{2}=\sum_{i=1}^{9} w_{2 i} * x_{2 i}
$$

Where $F_{2}$ is the value of docking precision, $w_{2 i}$ is the weight coefficient of $\mathrm{i}$ index, $x_{2 i}$ is the value of $\mathrm{i}$ index. The weight coefficients were analyzed by the entropy analysis method.

The Rendezvous process was divided into two parts, tracking control stage and accurate control stage. The model was:

$$
F_{3}=w_{A} * F_{A}+w_{B} * F_{B}
$$

Where $F_{3}$ is the value of whole Rendezvous process, $F_{A}$ and $F_{B}$ are the values of tracking control stage and accurate control stage, $w_{A}$ and $w_{B}$ : the weight coefficient of tracking control stage and accurate control stage.

The weight coefficient of tracking control stage and accurate control stage were analyzed by expert assign method. The values of tracking control stage and accurate control stage were obtained by the factor analysis method

\section{Validation Experiment}

\subsection{MCRVD Simulator}

For examine characteristics and regulations of MCRVD performance indexes, a ground RVD simulator was developed. This simulator can simulate the process of MCRVD in the last $150 \mathrm{~m}$. The simulator have human-machine interface that contain spacecraft cabin, seats, controller, Video display interface, Control dynamics module.[7]

For the experiment requirement, the design parameters of simulator can be changed, e.g. the installation position of seats, the contents, layout, target sign and referential ruler of display, etc.

\subsection{Experiment Design}

The experiment design three scheme distinguish from different task difficulty. The experiment aimed at the performance of three schemes. The parameters contain numerical value display type and assistant task in table. 3 . 
Table 3. Experiment scheme parameter

\begin{tabular}{ccc}
\hline Scheme name & $\begin{array}{c}\text { Numerical value } \\
\text { display type }\end{array}$ & Assistant task \\
\hline Scheme A & numerical value & - \\
Scheme B & Non-numerical value & - \\
Scheme C & Non-numerical value & $\begin{array}{c}\text { distinguish from sound } \\
\text { stimulation }\end{array}$ \\
\hline
\end{tabular}

The operators in the experiment were technicians from the Astronaut Research and Training Center of China. They included 25 males ranging from 22 to 40 years old. The operators all had at least a bachelor's degree and work experience related to manned spaceflight. Everyone has operated 3 times. After experiment, everyone was required to fill in the subjective post experiment questionnaire.

\subsection{Data Analysis}

Multiple comparisons of value between different scheme divisions were conducted by using One-way ANOVA. The result of the experiment showed in table 4.

Table 4. Multiple comparisons in One-way ANOVA

\begin{tabular}{lcc}
\hline Scheme I & Scheme J & Sig. \\
\hline Scheme A & Scheme B & 0.211 \\
Scheme A & Scheme C & 0.001 \\
Scheme B & Scheme C & 0.001 \\
\hline
\end{tabular}

Data Analysis and Results showed that: the value did significantly discrepancy between scheme A and scheme $C(p<0.05)$, and the same result between scheme $B$ and scheme $\mathrm{C}(\mathrm{p}<0.05)$. it is the same as the result of the subjective experiment questionnaire.

It is ensure that the model was validated.

\section{Conclusions}

The performance indexes of MCRVD show the characteristics and laws of stages. The process of MCRVD can be divided into three stages: tracking control (about more than $20 \mathrm{~m}$ distance), accurate control (less than $20 \mathrm{~m}$ distance) and docking stage $(0 \mathrm{~m})$. The performance indexes of tracking and accurate control show the relevance of characteristics, and the precision index of docking reflects the difficulty of control the direction of the spacecraft.

For that reason, several statistics techniques, such as the factor analysis method, the entropy analysis method etc, are utilized to analyze the weight coefficient of each performance index. After that we presented a novel multi-hierarchy integrated evaluation method, which includes four hierarchies: the performance of the tracking 
control, the performance of the accurate control, the precision of docking and the result of docking. Finally we utilized this method to analyze the human performance in MCRVD, which verifies the validity of our method.

Acknowledgements. This study is supported by The National Basic Research Program of China (973). (No.2011CB711000).

\section{References}

1. Zhang, Y.J., Xu, Y.Z., Li, Z.Z., Li, J., Wu, S.: Influence of Monitoring Method and Control Complexity on Operator Performance in Manually Controlled Spacecraft Rendezvous and Docking, vol. 5(12), pp. 619-624 (2008)

2. Carolina, L.L., Edward, V.B.: A Survey of Rendezvous and Docking Issues and Development. AAS-89-158 (1989)

3. Collins, D.J.: Fight Operations for Shuttle Rendezvous Navigation and Targeting. Proceeding IEEE (1984)

4. Fehse, W.: Rendezvous and Docking Technology Development for Future European Missions, vol. 9(1). ESA (1985)

5. Clandinon, B.: Detailed System Studies on RVD. N84-33428 (1984)

6. Eggleston, J.M.: A Study of the Optimum Velocity Change to Intercept and Rendezvous. NASA-TND-1029 (1962)

7. Nunamaker, R.R., Rowell, L.F.: Langley Research Center Resources and Need for Manned Space Operations Simulation. NASA Langley Research Center, Paper (1987) 